\title{
GENERALIZED WEAKLY $\alpha$-CONTRACTIVE MAPPINGS AND APPLICATIONS TO ORDINARY DIFFERENTIAL EQUATIONS
}

\author{
HUSEYIN ISIK AND DURAN TURKOGLU
}

Received 17 October, 2014

\begin{abstract}
In this paper, we introduce the notion of generalized weakly $\alpha$-contractive mappings and give some common fixed point results for this type of contraction. The presented theorems extend, generalize and improve many existing results in the literature. Two examples as well as an application to ordinary differential equations are also given in order to illustrate the effectiveness of the obtained results.
\end{abstract}

2010 Mathematics Subject Classification: 54H25; 47H10

Keywords: common fixed point, point of coincidence, generalized weakly $\alpha$-contraction, weakly compatible mappings, two point boundary value problem

\section{INTRODUCTION AND PRELIMINARIES}

Fixed point theory is one of the most powerful and effective tools in mathematics which has enormous applications within as well as outside mathematics. One of the most fundamental and the most widely applied result in metric fixed point theory is "Banach contraction principle" due to Banach [2]. The basic idea of this principle rests in the use of successive approximations to establish the existence and uniqueness of solution of an operator equation $f(x)=x$, particularly it can be employed to prove the existence of solution of differential or integral equations. Due to its applications in mathematics and other related disciplines, Banach contraction principle has been generalized in many directions (for example, see [1,3-21].

Khan et al. [10] introduced and employed the notion of altering distance function to obtain some interesting fixed point results in metric spaces. Note that altering distance functions are continuous whereas $\mathrm{Su}$ [21] defined generalized altering distance function, not necessarily continuos, as follows:

Definition 1 ([21]). A mapping $\psi:[0,+\infty) \rightarrow[0,+\infty)$ is called a generalized altering distance function if

(i) $\psi$ is non-decreasing,

(ii) $\psi(t)=0$ if and only if $t=0$. 
Recently, Samet et al. [20] took a new approach to the generalization of Banach contraction principle and introduced the notions of $\alpha$-admissible and $\alpha$ - $\psi$-contractive type mappings, while establishing various fixed point theorems for such mappings in the setting of complete metric spaces. After that, several authors considered the generalizations of this new approach (for example, see [3, 6, 7, 11-13, 16, 19]).

In this paper, we prove some common fixed point theorems for a class of generalized weakly $\alpha$-contractions in the setting of complete metric spaces. Our results improve and extend the results of Samet at al. [20] and many others. Also, two examples and an application to ordinary differential equations are considered in order to illustrate the effectiveness of the obtained results.

Definition 2 ([8]). A pair $(f, T)$ of self-mappings on a set $X$ is said to be weakly compatible if $f$ and $T$ commute at their coincidence point (i.e. $f T x=T f x, x \in X$ whenever $f x=T x$ ).

A point $y \in X$ is called a point of coincidence of self-mappings $f, g$ and $T$ on $X$ if there exists a point $x \in X$ such that $y=f x=g x=T x$. Also, an element $x \in X$ is called a common fixed point of $f, g$ and $T$, if $x=f x=g x=T x$.

The notation $\mathcal{C}(f, g, T)$ stands for the set of all coincidence points of $f, g$ and $T$. In the sequel, the letters $\mathbb{R}, \mathbb{R}^{+}$, and $\mathbb{N}$ will denote the set of all real numbers, the set of all non negative real numbers and the set of all natural numbers, respectively.

Definition 3 ([16]). Let $f, g$ and $T$ be self-mappings of a non-empty set $X$ and $\alpha: T X \times T X \rightarrow \mathbb{R}^{+}$be a given mapping. Then the pair $(f, g)$ is called $\alpha$-admissible with respect to $T$ (briefly, $(f, g)$ is $\alpha_{T}$-admissible) if for all $x, y \in X$,

$$
\alpha(T x, T y) \geq 1 \Longrightarrow \alpha(f x, g y) \geq 1 \text { and } \alpha(g x, f y) \geq 1 .
$$

Note that, if $f=g$, then $f$ is $T$ - $\alpha$-admissible defined in [12]. If $f=g$ and $T$ is the identity mapping, then $f$ is $\alpha$-admissible defined in [20].

Definition 4. Let $(X, d)$ be a metric space and $\alpha: X \times X \rightarrow \mathbb{R}^{+}$. We say that $X$ is $\alpha$-regular if, for every sequence $\left\{x_{n}\right\}$ in $X$ such that $\alpha\left(x_{n}, x_{n+1}\right) \geq 1$ for all $n$ and $x_{n} \rightarrow x \in X$ as $n \rightarrow+\infty$, then $\alpha\left(x_{n}, x\right) \geq 1$ for all $n$ and $\alpha(x, x) \geq 1$.

We set $\Psi=\left\{\psi: \mathbb{R}^{+} \rightarrow \mathbb{R}^{+}: \psi\right.$ is a generalized altering distance function $\}$ and $\Phi=\left\{\varphi: \mathbb{R}^{+} \rightarrow \mathbb{R}^{+}: \varphi\right.$ is a nondecreasing and right upper semi-continuous function with the condition $\psi(t)>\varphi(t)$ for all $t>0$ where $\psi \in \Psi$ \}.

Definition 5. Let $(X, d)$ be a metric space and, $f, g$ and $T$ be self-mappings on $X$ and $\alpha: T X \times T X \rightarrow \mathbb{R}^{+}$be a given function. Then, we say that the pair $(f, g)$ is a generalized weakly $\alpha_{T}$-contraction if, for all $x, y \in X$,

$$
\psi(\alpha(T x, T y) d(f x, g y)) \leq \varphi(M(x, y)),
$$

where $\psi \in \Psi, \varphi \in \Phi$ and

$M(x, y)=\max \{d(T x, T y), d(T x, f x), d(T y, g y),[d(T x, g y)+d(f x, T y)] / 2\}$. 


\section{MAIN RESULTS}

Our main result is the following.

Theorem 1. Let $f, g$ and $T$ be selfmaps of a complete metric space $(X, d)$ with $f X \subset T X, g X \subset T X$ and the pair $(f, g)$ be an $\alpha_{T}$-admissible and a generalized weakly $\alpha_{T}$-contraction. Assume that $T X$ is a closed subset of $X$ and that the following conditions are satisfied:

(i) there exists $x_{0} \in X$ such that $\alpha\left(T x_{0}, f x_{0}\right) \geq 1$;

(ii) $X$ is $\alpha$-regular and, for every sequence $\left\{x_{n}\right\}$ in $X$ such that $\alpha\left(x_{n}, x_{n+1}\right) \geq 1$, we have $\alpha\left(x_{m}, x_{n}\right) \geq 1$ for all $m, n \in \mathbb{N}$ with $m<n$;

(iii) either $\alpha(T u, T v) \geq 1$ or $\alpha(T v, T u) \geq 1$ whenever $u, v \in \mathcal{C}(f, g, T)$.

Then $f, g$ and $T$ have a unique point of coincidence in $X$. Moreover, if the pairs $(f, T)$ and $(g, T)$ are weakly compatible, then $f, g$ and $T$ have a unique common fixed point.

Proof. Let $x_{0} \in X$ such that $\alpha\left(T x_{0}, f x_{0}\right) \geq 1$. Since $f X \subset T X$, there exists an $x_{1} \in X$ such that $f x_{0}=T x_{1}$. Again since $g X \subset T X$, there exists an $x_{2} \in X$ such that $g x_{1}=T x_{2}$. Continuing this process, we can construct sequences $\left\{x_{n}\right\}$ and $\left\{y_{n}\right\}$ in $X$ defined by

$$
y_{2 n}=f x_{2 n}=T x_{2 n+1}, \quad y_{2 n+1}=g x_{2 n+1}=T x_{2 n+2}, \quad n \in \mathbb{N}_{0}
$$

where $\mathbb{N}_{0}=\mathbb{N} \cup\{0\}$. Since the pair $(f, g)$ is $\alpha_{T}$-admissible

$$
\alpha\left(T x_{0}, f x_{0}\right)=\alpha\left(T x_{0}, T x_{1}\right) \geq 1 \Longrightarrow \alpha\left(f x_{0}, g x_{1}\right) \geq 1 \text { and } \alpha\left(g x_{0}, f x_{1}\right) \geq 1 \text {, }
$$

which implies $\alpha\left(T x_{1}, T x_{2}\right) \geq 1$. Again

$$
\alpha\left(T x_{1}, T x_{2}\right) \geq 1 \Longrightarrow \alpha\left(f x_{1}, g x_{2}\right) \geq 1 \text { and } \alpha\left(g x_{1}, f x_{2}\right) \geq 1,
$$

and so $\alpha\left(T x_{2}, T x_{3}\right) \geq 1$. Inductively, we get

$$
\alpha\left(T x_{n}, T x_{n+1}\right) \geq 1, \quad n \in \mathbb{N}_{0} .
$$

Now, we show that $\left\{y_{n}\right\}$ is a Cauchy sequence in $X$. Consider the following two cases.

Case 1: If for some $n, y_{n}=y_{n+1}$ then $y_{n+1}=y_{n+2}$. If not, then for $n=2 m$ where $m \in \mathbb{N}_{0}$, we have

$$
\begin{aligned}
& M\left(x_{2 m+1}, x_{2 m+2}\right)=\max \left\{d\left(T x_{2 m+1}, T x_{2 m+2}\right), d\left(T x_{2 m+1}, f x_{2 m+1}\right),\right. \\
& d\left(T x_{2 m+2}, g x_{2 m+2}\right), \\
&= {\left.\left[d\left(T x_{2 m+1}, g x_{2 m+2}\right)+d\left(f x_{2 m+1}, T x_{2 m+2}\right)\right] / 2\right\} } \\
&=\max \left\{d\left(y_{2 m}, y_{2 m+1}\right), d\left(y_{2 m}, y_{2 m+1}\right), d\left(y_{2 m+1}, y_{2 m+2}\right),\right. {\left.\left[d\left(y_{2 m}, y_{2 m+2}\right)+d\left(y_{2 m+1}, y_{2 m+1}\right)\right] / 2\right\} } \\
& \leq \max \left\{0, d\left(y_{2 m+1}, y_{2 m+2}\right), \frac{1}{2} d\left(y_{2 m+1}, y_{2 m+2}\right)\right\}
\end{aligned}
$$




$$
=d\left(y_{2 m+1}, y_{2 m+2}\right) \text {. }
$$

By (1.1)

$$
\begin{aligned}
\psi\left(d\left(y_{2 m+1}, y_{2 m+2}\right)\right) & =\psi\left(d\left(f x_{2 m+1}, g x_{2 m+2}\right)\right) \\
& \leq \psi\left(\alpha\left(T x_{2 m+1}, T x_{2 m+2}\right) d\left(f x_{2 m+1}, g x_{2 m+2}\right)\right) \\
& \leq \varphi\left(M\left(x_{2 m+1}, x_{2 m+2}\right)\right) \\
& \leq \varphi\left(d\left(y_{2 m+1}, y_{2 m+2}\right)\right),
\end{aligned}
$$

which is a contradiction. Hence we must have $y_{n+1}=y_{n+2}$, when $n$ is even. By proceeding in this way, we obtain $y_{n+k}=y_{n}$ for all $k \in \mathbb{N}$. Similar is the case when $n$ is odd. Thus, we conclude that $\left\{y_{n}\right\}$ is a Cauchy sequence.

Case 2: Suppose that $y_{n} \neq y_{n+1}$ for all $n \in \mathbb{N}_{0}$. Then, putting $x=x_{2 n}$ and $y=x_{2 n+1}$ in (1.1) and, using (2.1) and (2.2), we obtain

$$
\begin{aligned}
\psi\left(d\left(y_{2 n}, y_{2 n+1}\right)\right) & =\psi\left(d\left(f x_{2 n}, g x_{2 n+1}\right)\right) \\
& \leq \psi\left(\alpha\left(T x_{2 n}, T x_{2 n+1}\right) d\left(f x_{2 n}, g x_{2 n+1}\right)\right) \\
& \leq \varphi\left(M\left(x_{2 n}, x_{2 n+1}\right)\right),
\end{aligned}
$$

where

$$
\begin{aligned}
M\left(x_{2 n}, x_{2 n+1}\right)= & \max \left\{d\left(T x_{2 n}, T x_{2 n+1}\right), d\left(T x_{2 n}, f x_{2 n}\right), d\left(T x_{2 n+1}, g x_{2 n+1}\right),\right. \\
= & {\left.\left[d\left(T x_{2 n}, g x_{2 n+1}\right)+d\left(f x_{2 n}, T x_{2 n+1}\right)\right] / 2\right\} } \\
= & \max \left\{d\left(y_{2 n-1}, y_{2 n}\right), d\left(y_{2 n-1}, y_{2 n}\right), d\left(y_{2 n}, y_{2 n+1}\right),\right. \\
\leq & {\left.\left[d\left(y_{2 n-1}, y_{2 n+1}\right)+d\left(y_{2 n}, y_{2 n}\right)\right] / 2\right\} } \\
& \max \left\{d\left(y_{2 n-1}, y_{2 n}\right), d\left(y_{2 n}, y_{2 n+1}\right),\right. \\
= & \left.\quad\left[d\left(y_{2 n-1}, y_{2 n}\right)+d\left(y_{2 n}, y_{2 n+1}\right)\right] / 2\right\} \\
= & \max \left\{d\left(y_{2 n-1}, y_{2 n}\right), d\left(y_{2 n}, y_{2 n+1}\right)\right\} .
\end{aligned}
$$

If $M\left(x_{2 n}, x_{2 n+1}\right)=d\left(y_{2 n}, y_{2 n+1}\right)$, by (2.3) and using the properties of $\psi$ and $\varphi$, we have

$$
\psi\left(d\left(y_{2 n}, y_{2 n+1}\right)\right) \leq \varphi\left(d\left(y_{2 n}, y_{2 n+1}\right)\right),
$$

and so $d\left(y_{2 n}, y_{2 n+1}\right)=0$, which is a contradiction. Thus $M\left(x_{2 n}, x_{2 n+1}\right)=$ $d\left(y_{2 n-1}, y_{2 n}\right)>0$, then from (2.3), we get

$$
\psi\left(d\left(y_{2 n}, y_{2 n+1}\right)\right) \leq \varphi\left(d\left(y_{2 n-1}, y_{2 n}\right)\right) .
$$

Putting $x=x_{2 n+1}$ and $y=x_{2 n+2}$ in (1.1) and following arguing similar to those given above, we obtain

$$
\psi\left(d\left(y_{2 n+1}, y_{2 n+2}\right)\right) \leq \varphi\left(d\left(y_{2 n}, y_{2 n+1}\right)\right) .
$$

From (2.4) and (2.5), we conclude that

$$
\psi\left(d\left(y_{n}, y_{n+1}\right)\right) \leq \varphi\left(d\left(y_{n-1}, y_{n}\right)\right) .
$$


Using the properties of $\psi$ and $\varphi$ in (2.6) we deduce that $d\left(y_{n}, y_{n+1}\right) \leq d\left(y_{n-1}, y_{n}\right)$, that is, the sequence $\left\{d\left(y_{n}, y_{n+1}\right)\right\}$ is decreasing. Hence, there exists $r \geq 0$ such that $\lim _{n \rightarrow \infty} d\left(y_{n}, y_{n+1}\right)=r$. We claim that $r=0$. Suppose, on the contrary, that $r>0$. Then, letting $n \rightarrow \infty$ in (2.6), we have

$$
\begin{aligned}
\psi(r) & \leq \lim _{n \rightarrow \infty} \psi\left(d\left(y_{n}, y_{n+1}\right)\right) \\
& \leq \lim _{n \rightarrow \infty} \varphi\left(d\left(y_{n-1}, y_{n}\right)\right) \leq \varphi(r),
\end{aligned}
$$

a contradiction and hence $r=0$, that is

$$
\lim _{n \rightarrow \infty} d\left(y_{n}, y_{n+1}\right)=0 .
$$

Now, we prove that $\left\{y_{n}\right\}$ is a Cauchy sequence. To this end, it is sufficient to verify that $\left\{y_{2 n}\right\}$ is a Cauchy sequence. Suppose, to the contrary, that $\left\{y_{2 n}\right\}$ is not a Cauchy sequence. Then, there exists an $\varepsilon>0$ for which we can find two subsequences $\left\{y_{2 m_{k}}\right\}$ and $\left\{y_{2 n_{k}}\right\}$ of $\left\{y_{2 n}\right\}$ such that $n_{k}$ is the smallest index for which $n_{k}>m_{k}>k$ and

$$
d\left(y_{2 m_{k}}, y_{2 n_{k}}\right) \geq \varepsilon \text { and } d\left(y_{2 m_{k}}, y_{2 n_{k}-1}\right)<\varepsilon .
$$

Using the triangular inequality and (2.8), we get

$$
\begin{aligned}
\varepsilon & \leq d\left(y_{2 m_{k}}, y_{2 n_{k}}\right) \leq d\left(y_{2 n_{k}}, y_{2 n_{k}-1}\right)+d\left(y_{2 n_{k}-1}, y_{2 m_{k}}\right) \\
& <d\left(y_{2 n_{k}}, y_{2 n_{k}-1}\right)+\varepsilon .
\end{aligned}
$$

Taking $k \rightarrow \infty$ in the above inequality and using (2.7), we obtain

$$
\lim _{k \rightarrow \infty} d\left(y_{2 m_{k}}, y_{2 n_{k}}\right)=\varepsilon
$$

Again, using the triangular inequality, we deduce

$$
\left|d\left(y_{2 m_{k}}, y_{2 n_{k}+1}\right)-d\left(y_{2 m_{k}}, y_{2 n_{k}}\right)\right| \leq d\left(y_{2 n_{k}}, y_{2 n_{k}+1}\right) .
$$

Letting $k \rightarrow \infty$ in the above inequality and using (2.7) and (2.9), we have

$$
\lim _{k \rightarrow \infty} d\left(y_{2 m_{k}}, y_{2 n_{k}+1}\right)=\varepsilon .
$$

Similarly, it is easy to show that

$$
\lim _{k \rightarrow \infty} d\left(y_{2 m_{k}-1}, y_{2 n_{k}}\right)=\lim _{k \rightarrow \infty} d\left(y_{2 m_{k}-1}, y_{2 n_{k}+1}\right)=\varepsilon .
$$

By (ii) and (2.2), we know that $\alpha\left(T x_{2 m_{k}}, T x_{2 n_{k}+1}\right) \geq 1$ for $n_{k}>m_{k}$. Then, from (1.1) with $x=x_{2 m_{k}}$ and $y=x_{2 n_{k}+1}$, we get

$$
\begin{aligned}
\psi\left(d\left(y_{2 m_{k}}, y_{2 n_{k}+1}\right)\right) & =\psi\left(d\left(f x_{2 m_{k}}, g x_{2 n_{k}+1}\right)\right) \\
& \leq \psi\left(\alpha\left(T x_{2 m_{k}}, T x_{2 n_{k}+1}\right) d\left(f x_{2 m_{k}}, g x_{2 n_{k}+1}\right)\right) \\
& \leq \varphi\left(M\left(x_{2 m_{k}}, x_{2 n_{k}+1}\right)\right),
\end{aligned}
$$


where

$$
\begin{aligned}
& M\left(x_{2 m_{k}}, x_{2 n_{k}+1}\right)=\max \left\{d\left(T x_{2 m_{k}}, T x_{2 n_{k}+1}\right), d\left(T x_{2 m_{k}}, f x_{2 m_{k}}\right),\right. \\
& d\left(T x_{2 n_{k}+1}, g x_{2 n_{k}+1}\right), \\
& {\left.\left[d\left(T x_{2 m_{k}}, g x_{2 n_{k}+1}\right)+d\left(f x_{2 m_{k}}, T x_{2 n_{k}+1}\right)\right] / 2\right\} } \\
&=\max \left\{d\left(y_{2 m_{k}-1}, y_{2 n_{k}}\right), d\left(y_{2 m_{k}-1}, y_{2 m_{k}}\right), d\left(y_{2 n_{k}}, y_{2 n_{k}+1}\right),\right. \\
& {\left.\left[d\left(y_{2 m_{k}-1}, y_{2 n_{k}+1}\right)+d\left(y_{2 m_{k}}, y_{2 n_{k}}\right)\right] / 2\right\} }
\end{aligned}
$$

Now, using (2.7), (2.9), (2.10) and (2.11) and taking $k \rightarrow \infty$ in (2.12), we deduce

$$
\begin{aligned}
\psi(\varepsilon) & \leq \lim _{k \rightarrow \infty} \psi\left(d\left(y_{2 m_{k}}, y_{2 n_{k}+1}\right)\right) \\
& \leq \lim _{k \rightarrow \infty} \varphi\left(M\left(x_{2 m_{k}}, x_{2 n_{k}+1}\right)\right) \\
& \leq \varphi(\max \{\varepsilon, 0,0, \varepsilon\})=\varphi(\varepsilon),
\end{aligned}
$$

which implies that $\varepsilon=0$, a contradiction with $\varepsilon>0$. Thus, $\left\{y_{2 n}\right\}$ is a Cauchy sequence in $X$ and hence $\left\{y_{n}\right\}$ is a Cauchy sequence. From the completeness of $(X, d)$, there exists $z \in X$ such that

$$
\lim _{n \rightarrow \infty} y_{n}=z
$$

From (2.1) and (2.13), we obtain

$$
f x_{2 n} \rightarrow z, \quad T x_{2 n+1} \rightarrow z, \quad g x_{2 n+1} \rightarrow z, \quad T x_{2 n+2} \rightarrow z .
$$

Since $T X$ is closed, by (2.14), $z \in T X$. Therefore, there exists $u \in X$ such that $T u=$ $z$. Since $X$ is $\alpha$-regular, then $\alpha\left(y_{n}, z\right) \geq 1$ for all $n \in \mathbb{N}_{0}$ and $\alpha(z, z) \geq 1$. Now, applying inequality (1.1) with $x=x_{2 n}$ and $y=u$, we have

$$
\begin{aligned}
\psi\left(d\left(y_{2 n+1}, g u\right)\right) & =\psi\left(d\left(f x_{2 n+1}, g u\right)\right) \\
& \leq \psi\left(\alpha\left(T x_{2 n+1}, T u\right) d\left(f x_{2 n+1}, g u\right)\right) \\
& \leq \varphi\left(M\left(x_{2 n+1}, u\right)\right)
\end{aligned}
$$

where

$$
\begin{gathered}
M\left(x_{2 n+1}, u\right)=\max \left\{d\left(T x_{2 n+1}, T u\right), d\left(T x_{2 n+1}, f x_{2 n+1}\right), d(T u, g u),\right. \\
\left.\left[d\left(T x_{2 n+1}, g u\right)+d\left(f x_{2 n+1}, T u\right)\right] / 2\right\} .
\end{gathered}
$$

Taking $k \rightarrow \infty$ in the inequality (2.16), we get

$$
\begin{aligned}
\psi(d(z, g u)) & \leq \lim _{n \rightarrow \infty} \psi\left(d\left(y_{2 n+1}, g u\right)\right) \\
& \leq \lim _{n \rightarrow \infty} \varphi\left(M\left(x_{2 n+1}, u\right)\right) \\
& \leq \varphi\left(\max \left(0,0, d(z, g u), \frac{d(z, g u)}{2}\right)\right) \\
& =\varphi(d(z, g u)),
\end{aligned}
$$


which implies $d(z, g u)=0$, that is, $z=g u$. Again, by (1.1), we obtain

$$
\begin{aligned}
\psi(d(f u, z)) & =\psi(d(f u, g u)) \\
& \leq \psi(\alpha(T u, T u) d(f u, g u)) \\
& \leq \varphi(M(u, u))
\end{aligned}
$$

where

$$
\begin{aligned}
M(u, u) & =\max \{d(T u, T u), d(T u, f u), d(T u, g u),[d(T u, g u)+d(f u, T u)] / 2\} \\
& =\max \left\{0, d(z, f u), 0, \frac{d(f u, z)}{2}\right\} \\
& =d(f u, z) .
\end{aligned}
$$

From (2.17), we have

$$
\psi(d(f u, z)) \leq \varphi(d(f u, z))
$$

which implies $d(f u, z)=0$, that is, $z=f u$. Thus, we deduce that

$$
z=f u=g u=T u,
$$

and so, $z$ is a point of coincidence of $f, g$ and $T$. The uniqueness of the point of coincidence is a consequence of the conditions (1.1) and (i i i ), and so we omit the details.

By (2.18) and using weakly compatibility of the pairs $(f, T)$ and $(g, T)$, we obtain

$$
f z=f T u=T f u=T z \quad \text { and } \quad g z=g T u=T g u=T z,
$$

and so $f z=g z=T z$. Uniqueness of the point of coincidence implies $z=f z=$ $g z=T z$, that is, $z$ is a unique common fixed point of $f, g$ and $T$.

Example 1. Let $X=\mathbb{R}^{+}$with the usual metric $d(x, y)=|x-y|$ for all $x, y \in X$ and $\psi, \varphi: \mathbb{R}^{+} \rightarrow \mathbb{R}^{+}$be defined by $\psi(t)=t$ and $\varphi(t)=\frac{t}{2}$. Define the mappings $f, g$ and $T$ on $X$ by

$$
\begin{gathered}
f x=\left\{\begin{array}{cl}
\frac{x}{6}, & x \in[0,1] \\
3 x, & x>1,
\end{array} \quad g x=\left\{\begin{array}{cc}
\frac{x}{8}, & x \in[0,1] \\
4 x, & x>1,
\end{array}\right.\right. \\
T x=\left\{\begin{array}{cc}
\frac{x}{2}, & x \in[0,1] \\
2 x, & x>1 .
\end{array}\right.
\end{gathered}
$$

Then, it is clear that $f X \subset T X$ and $g X \subset T X$. Also, we define the mapping $\alpha$ : $T X \times T X \rightarrow \mathbb{R}^{+}$by

$$
\alpha(x, y)= \begin{cases}1, & \text { if } x, y \in\left[0, \frac{1}{2}\right] \\ 0, & \text { otherwise. }\end{cases}
$$

Now, let $x, y \in X$ such that $\alpha(T x, T y) \geq 1$. Then $T x, T y \in\left[0, \frac{1}{2}\right]$ and this implies that $x, y \in[0,1]$. By the definitions of $f, g$ and $\alpha$, we have $f x, g y \in\left[0, \frac{1}{2}\right]$ and $g x, f y \in\left[0, \frac{1}{2}\right]$ which implies that

$$
\alpha(f x, g y)=1 \quad \text { and } \quad \alpha(g x, f y)=1,
$$


that is, $(f, g)$ is $\alpha_{T}$-admissible. Moreover, the condition $\alpha\left(T x_{0}, f x_{0}\right) \geq 1$ is satisfied with $x_{0}=0$.

Let $\left\{x_{n}\right\}$ be a sequence in $X$ such that $\alpha\left(x_{n}, x_{n+1}\right) \geq 1$ for all $n \in \mathbb{N}_{0}$. Then, $x_{n} \in\left[0, \frac{1}{2}\right]$ for all $n \in \mathbb{N}_{0}$. Thus, $x_{m} \in\left[0, \frac{1}{2}\right]$ for all $m, n \in \mathbb{N}$ with $m<n$, and so $\alpha\left(x_{m}, x_{n}\right) \geq 1$.

Again, let $\left\{x_{n}\right\}$ be a sequence in $X$ such that $\alpha\left(x_{n}, x_{n+1}\right) \geq 1$ for all $n \in \mathbb{N}_{0}$ and $x_{n} \rightarrow x$ as $n \rightarrow+\infty$. Then, $x_{n} \in\left[0, \frac{1}{2}\right]$ for all $n \in \mathbb{N}_{0}$, and so $x \in\left[0, \frac{1}{2}\right]$ as $x_{n} \rightarrow x$. Therefore, $\alpha\left(x_{n}, x\right) \geq 1$ for all $n \in \mathbb{N}_{0}$ and $\alpha(x, x) \geq 1$. This means that $X$ is $\alpha$-regular.

Now, we have to check the validity of contractive condition (1.1) only when $\alpha(T x, T y) \geq 1$, since the result is clear in other cases. Then, for all $x, y \in[0,1]$, we get

$$
\begin{aligned}
\psi(\alpha(T x, T y) d(f x, g y)) & =\psi(d(f x, g y)) \\
& =\left|\frac{4 x-3 y}{24}\right| \leq \frac{x}{6} \\
& =\frac{1}{2}|T x-f x| \leq \frac{1}{2} M(x, y) \\
& =\varphi(M(x, y)) .
\end{aligned}
$$

Obviously, the assumption (i i i ) of Theorem 1 is satisfied. Consequently, all conditions of Theorem 1 hold, and hence $f, g$ and $T$ have a unique common fixed point which is 0 .

If we choose $f=g$ in Theorem 1 , we have the following result.

Corollary 1. Let $f$ and $T$ be selfmaps of a complete metric space $(X, d)$ with $f X \subset T X$ and $f$ be a $T$ - $\alpha$-admissible mapping. Suppose that $T X$ is a closed subset of $X$ such that

$$
\psi(\alpha(T x, T y) d(f x, f y)) \leq \varphi(M(x, y)),
$$

for all $x, y \in X$, where $\psi \in \Psi, \varphi \in \Phi$ and

$$
M(x, y)=\max \{d(T x, T y), d(T x, f x), d(T y, f y),[d(T x, f y)+d(f x, T y)] / 2\} .
$$

Assume also that the following conditions are satisfied:

(i) there exists $x_{0} \in X$ such that $\alpha\left(T x_{0}, f x_{0}\right) \geq 1$;

(ii) $X$ is $\alpha$-regular and, for every sequence $\left\{x_{n}\right\}$ in $X$ such that $\alpha\left(x_{n}, x_{n+1}\right) \geq 1$, we have $\alpha\left(x_{m}, x_{n}\right) \geq 1$ for all $m, n \in \mathbb{N}$ with $m<n$;

(iii) either $\alpha(T u, T v) \geq 1$ or $\alpha(T v, T u) \geq 1$ whenever $u, v \in \mathcal{C}(f, T)$.

Then $f$ and $T$ have a unique point of coincidence in $X$. Moreover, if the pair $(f, T)$ is weakly compatible, then $f$ and $T$ have a unique common fixed point.

If we choose $T=I_{X}$ and replace $d(x, y)$ with $M(x, y)$ in Corollary 1 , we have the following result. 
Corollary 2. Let $(X, d)$ be a complete metric space, $f$ be a self-mapping on $X$ and $f$ be an $\alpha$-admissible. Suppose that

$$
\psi(\alpha(x, y) d(f x, f y)) \leq \varphi(d(x, y)),
$$

for all $x, y \in X$, where $\psi \in \Psi$ and $\varphi \in \Phi$. Assume also that the following conditions are satisfied:

(i) there exists $x_{0} \in X$ such that $\alpha\left(x_{0}, f x_{0}\right) \geq 1$;

(ii) $X$ is $\alpha$-regular and, for every sequence $\left\{x_{n}\right\}$ in $X$ such that $\alpha\left(x_{n}, x_{n+1}\right) \geq 1$, we have $\alpha\left(x_{m}, x_{n}\right) \geq 1$ for all $m, n \in \mathbb{N}$ with $m<n$;

(iii) either $\alpha(u, v) \geq 1$ or $\alpha(v, u) \geq 1$ whenever $u=f u$ and $v=f v$.

Then $f$ has a unique fixed point.

If we take $\psi(t)=t$ in Corollary 2, we have the following corollary.

Corollary 3 ([20]). Let $(X, d)$ be a complete metric space, $f$ be a self-mapping on $X$ and $f$ be an $\alpha$-admissible. Suppose that

$$
\alpha(x, y) d(f x, f y) \leq \varphi(d(x, y)),
$$

for all $x, y \in X$, where $\varphi \in \Phi$. Assume also that the following conditions are satisfied:

(i) there exists $x_{0} \in X$ such that $\alpha\left(x_{0}, f x_{0}\right) \geq 1$;

(ii) $X$ is $\alpha$-regular and, for every sequence $\left\{x_{n}\right\}$ in $X$ such that $\alpha\left(x_{n}, x_{n+1}\right) \geq 1$, we have $\alpha\left(x_{m}, x_{n}\right) \geq 1$ for all $m, n \in \mathbb{N}$ with $m<n$;

(iii) either $\alpha(u, v) \geq 1$ or $\alpha(v, u) \geq 1$ whenever $u=f u$ and $v=f v$.

Then $f$ has a unique fixed point.

From Theorem 1, if $\alpha: T X \times T X \rightarrow \mathbb{R}^{+}$is a function such that $\alpha(T x, T y)=1$ for all $x, y \in X$, we deduce the following theorem.

Theorem 2. Let $f, g$ and $T$ be selfmaps of a complete metric space $(X, d)$ with $f X \subset T X$ and $g X \subset T X$. Assume that $T X$ is a closed subset of $X$ such that

$$
\psi(d(f x, g y)) \leq \varphi(M(x, y)),
$$

for all $x, y \in X$, where $\psi \in \Psi, \varphi \in \Phi$ and

$$
\begin{aligned}
& M(x, y) \\
& \quad=\max \{d(T x, T y), d(T x, f x), d(T y, g y),[d(T x, g y)+d(f x, T y)] / 2\} .
\end{aligned}
$$

Then $f, g$ and $T$ have a unique point of coincidence in $X$. Moreover, if the pairs $(f, T)$ and $(g, T)$ are weakly compatible, then $f, g$ and $T$ have a unique common fixed point.

The existence of fixed points of nonlinear contraction mappings in metric spaces endowed with a partial ordering has been considered recently by Ran and Reurings [17] in order to obtain a solution of a matrix equation in 2004. Nieto and Lopez [15] 
extended the results in [17] by removing the continuity condition of the mapping and applied their result to get a solution of a boundary value problem.

Let $X$ be a non-empty set. If $d$ is a complete metric on $X$ and $\preceq$ is a partial order on the set $X$, then $(X, d, \preceq)$ is called complete partially ordered metric space. Let $(X, \preceq)$ be a partially ordered set and $f, T: X \rightarrow X$ be two mappings. $f$ is called a $T$-nondecreasing mapping if $f x \preceq f y$ whenever $T x \preceq T y$ for all $x, y \in X$.

From Corollary 1, in the setting of complete partially ordered metric spaces, we obtain the following theorem.

Theorem 3. Let $(X, d, \preceq)$ be a complete partially ordered metric space, $f$ and $T$ be self-mappings on $X$ with $f X \subset T X$, and $f$ be a T-nondecreasing. Suppose that $T X$ is a closed subset of $X$ such that

$$
\psi(d(f x, f y)) \leq \varphi(M(x, y)),
$$

for all $x, y \in X$ such that $T x \preceq T y$, where $\psi \in \Psi, \varphi \in \Phi$ and

$M(x, y)=\max \{d(T x, T y), d(T x, f x), d(T y, f y),[d(T x, f y)+d(f x, T y)] / 2\}$.

Assume also that the following conditions are satisfied:

(i) there exists $x_{0} \in X$ such that $T x_{0} \preceq f x_{0}$;

(ii) if $\left\{x_{n}\right\}$ is a sequence in $X$ such that $x_{n} \preceq x_{n+1}$ for all $n \in \mathbb{N}_{0}$ and $x_{n} \rightarrow x$, then $x_{n} \preceq x$ for all $n \in \mathbb{N}_{0}$;

(iii) for every sequence $\left\{x_{n}\right\}$ in $X$ such that $x_{n} \preceq x_{n+1}$ for all $n \in \mathbb{N}_{0}$, we have $x_{m} \preceq x_{n}$ for all $m, n \in \mathbb{N}$ with $m<n$;

(iv) for all $u, v \in X$ such that $T u=f u$ and $T v=f v$, then $T u$ and $T v$ are comparable.

Then $f$ and $T$ have a unique point of coincidence in X. Moreover, if the pair $(f, T)$ is weakly compatible, then $f$ and $T$ have a unique common fixed point.

Proof. Define the function $\alpha: T X \times T X \rightarrow \mathbb{R}^{+}$by

$$
\alpha(x, y)= \begin{cases}1, & \text { if } x \preceq y \\ 0, & \text { otherwise. }\end{cases}
$$

Then, by (2.23), we have $\psi(\alpha(T x, T y) d(f x, f y)) \leq \varphi(M(x, y))$ for all $x, y \in$ $X$ with $T x \preceq T y$ and so (2.19) is satisfied.

Now, let $x, y \in X$ such that $\alpha(T x, T y) \geq 1$, then $T x \preceq T y$. Since $f$ is $T$-nondecreasing, we have $f x \preceq f y$, which gives us that $\alpha(f x, f y) \geq 1$. Then $f$ is $T$ - $\alpha$ admissible.

Let $\left\{x_{n}\right\}$ be a sequence in $X$ such that $\alpha\left(x_{n}, x_{n+1}\right) \geq 1$ for all $n \in \mathbb{N}_{0}$ and $x_{n} \rightarrow x$ as $n \rightarrow \infty$. Then $x_{n}, x_{n+1} \in T X$ and $x_{n} \preceq x_{n+1}$ for all $n \in \mathbb{N}_{0}$. Since $T X$ is closed, we deduce that $x \in T X$. From (ii), $x_{n} \preceq x$ for all $n \in \mathbb{N}_{0}$, and so $\alpha\left(x_{n}, x\right) \geq 1$ for all $n \in \mathbb{N}_{0}$. Also, because of $x \preceq x$ for all $x \in X$, we deduce that $\alpha(x, x) \geq 1$. Hence, $X$ is $\alpha$-regular. Similarly, by $(i i i)$, it is easy to see that $\alpha\left(x_{m}, x_{n}\right) \geq 1$ for all $m, n \in \mathbb{N}$ with $m<n$. Thus, $(i i)$ of Corollary 1 is satisfied. The same considerations show that 
(i) and (iv) of this theorem imply (i) and (iii) of Corollary 1. Therefore, all the hypotheses of Corollary 1 are satisfied, and so $f$ and $T$ have a unique common fixed point.

\section{APPLICATION: EXISTENCE THEOREM FOR A SOLUTION OF AN ORDINARY DIFFERENTIAL EQUATION}

We consider the following two-point boundary value problem of second order differential equation:

$$
\left\{\begin{aligned}
-\frac{d^{2} x}{d t^{2}} & =f(t, x(t)), \quad t \in[0,1] \\
x(0) & =x(1)=0,
\end{aligned}\right.
$$

where $f:[0,1] \times \mathbb{R} \rightarrow \mathbb{R}$ is a continuous function.

Let $X:=C(I)(I=[0,1])$ be the space of all continuous functions defined on $I$, and let

$$
d(x, y)=\sup _{t \in I}|x(t)-y(t)|,
$$

for all $x, y \in X$. Then, $(X, d)$ is a complete metric space.

The boundary value problem (3.1) can be written as the integral equation

$$
x(t)=\int_{0}^{1} G(t, s) f(s, x(s)) d s, \text { for all } t \in I,
$$

where $G(t, s)$ is the Green function given by

$$
G(t, s)= \begin{cases}t(1-s), & 0 \leq t \leq s \leq 1 \\ s(1-t), & 0 \leq s \leq t \leq 1 .\end{cases}
$$

Define the operator $F: X \rightarrow X$ by

$$
F x(t)=\int_{0}^{1} G(t, s) f(s, x(s)) d s, \quad \text { for all } t \in I .
$$

Then, the problem (3.1) is equivalent to find $x^{*} \in X$ that is a fixed point of $F$.

We consider the following conditions.

(i) there exists a function $\xi: X^{2} \rightarrow \mathbb{R}$ such that if $\xi(x, y) \geq 0$ for all $x, y \in X$, then we have

$$
[f(t, x)-f(t, y)]^{2} \leq 48 \theta(|x-y|),
$$

where $\theta: \mathbb{R}^{+} \rightarrow \mathbb{R}^{+}$is a nondecreasing and right upper semi-continuous function with $\theta(0)=0$ and $\theta(t)<t^{2}$ for all $t>0$;

(ii) there exists $x_{0} \in X$ such that $\xi\left(x_{0}, F x_{0}\right) \geq 0$;

(iii) for all $x, y \in X$, if $\xi(x, y) \geq 0$ implies $\xi(F x, F y) \geq 0$;

(iv) if $\left\{x_{n}\right\}$ is a sequence in $X$ such that $x_{n} \rightarrow x \in X$ and $\xi\left(x_{n}, x_{n+1}\right) \geq 0$ for all $n \in \mathbb{N}_{0}$, then $\xi\left(x_{n}, x\right) \geq 0$ for all $n \in \mathbb{N}_{0}$ and $\xi(x, x) \geq 0$;

(v) if $\left\{x_{n}\right\}$ is a sequence in $X$ such that $\xi\left(x_{n}, x_{n+1}\right) \geq 0$ for all $n \in \mathbb{N}_{0}$, then $\xi\left(x_{m}, x_{n}\right) \geq 0$ for all $m, n \in \mathbb{N}$ with $m<n$. 
Theorem 4. Suppose that conditions $(i)-(v)$ are satisfied. Then (3.1) has at least one solution $x^{*} \in X$.

Proof. Let $x, y \in X$ such that $\xi(x(t), y(t)) \geq 0$ for all $t \in I$. From condition $(i)$ and using Cauchy-Schwarz inequality, we have

$$
\begin{aligned}
{[F x(t)-F y(t)]^{2} } & =\left(\int_{0}^{1} G(t, s)[f(s, x(s))-f(s, y(s))] d s\right)^{2} \\
& \leq \int_{0}^{1} G(t, s)^{2} d s \int_{0}^{1}[f(s, x(s))-f(s, y(s))]^{2} d s \\
& \leq 48 \int_{0}^{1} G(t, s)^{2} d s \int_{0}^{1} \theta(|x(s)-y(s)|) d s \\
& \leq 48\left(\sup _{t \in I} \int_{0}^{1} G(t, s)^{2} d s\right) \int_{0}^{1} \theta(|x(s)-y(s)|) d s \\
& \leq 48\left(\sup _{t \in I} \int_{0}^{1} G(t, s)^{2} d s\right) \theta(d(x, y)) \\
& \leq \theta(d(x, y)),
\end{aligned}
$$

It is easy to verify that $\int_{0}^{1} G(t, s)^{2} d s=-\frac{2 t^{3}}{3}+\frac{t^{4}}{3}+\frac{t^{2}}{3}$ for all $t \in I$, which implies that $\sup _{t \in I} \int_{0}^{1} G(t, s)^{2} d s=\frac{1}{48}$.

By (3.2), we have

$$
\left(\sup _{t \in I}|F x(t)-F y(t)|\right)^{2} \leq \theta(d(x, y)) .
$$

Putting $\psi(t)=t^{2}$ and $\varphi(t)=\theta(t)$, we obtain

$$
\psi(d(F x, F y)) \leq \varphi(d(x, y))
$$

for all $x, y \in X$ such that $\xi(x(t), y(t)) \geq 0$ for all $t \in I$.

Define the function $\alpha: X \times X \rightarrow \mathbb{R}^{+}$by

$$
\alpha(x, y)= \begin{cases}1, & \text { if } \xi(x(t), y(t)) \geq 0, t \in I \\ 0, & \text { otherwise. }\end{cases}
$$

Then, for all $x, y \in X$, we deduce

$$
\psi(\alpha(x, y) d(F x, F y)) \leq \varphi(d(x, y)) .
$$

It easily shows that all the hypotheses of Corollary 2 are satisfied. Therefore, $F$ has a fixed point in $X$, that is, there exists $x^{*} \in X$ such that $x^{*}=F x^{*}$, and so $x^{*}$ is a solution to (3.1).

We now give an existence and uniqueness theorem for (3.1) under different conditions. 
Theorem 5. Consider problem (3.1) with $f: I \times \mathbb{R} \rightarrow \mathbb{R}$ is a continuous, nondecreasing with respect to the second variable and $f(t, 0) \geq 0$ for all $t \in I$. Suppose that there exists the function $\theta$ as in the condition ( $i$ ) of Theorem 4 such that for all $x, y \in X$ with $x \preceq y$

$$
[f(t, x)-f(t, y)]^{2} \leq 48 \theta(|x-y|),
$$

where $x \preceq y \Longleftrightarrow x(s) \leq y(s)$ for all $s \in I$. Then (3.1) has a unique solution.

Proof. Define $\alpha: X \times X \rightarrow \mathbb{R}^{+}$by

$$
\alpha(x, y)= \begin{cases}1, & \text { if } x \preceq y \\ 0, & \text { otherwise }\end{cases}
$$

If $\alpha(x, y) \geq 1$, then $x \preceq y$ and so $x(s) \leq y(s)$ for all $s \in I$. Since $f$ is nondecreasing with respect to the second variable, we get

$$
\begin{aligned}
F x(t) & =\int_{0}^{1} G(t, s) f(s, x(s)) d s \\
& \leq \int_{0}^{1} G(t, s) f(s, y(s)) d s=F y(t)
\end{aligned}
$$

for all $t \in I$. Hence, $F x \preceq F y$ and so $\alpha(F x, F y) \geq 1$. This means that $F$ is $\alpha$ admissible.

By a similar procedure as in Theorem 4 and using the inequality (3.3), for all $x, y \in X$ with $\alpha(x, y) \geq 1$, we obtain

$$
\psi(\alpha(x, y) d(F x, F y)) \leq \varphi(d(x, y)) .
$$

Also, since $f(s, 0) \geq 0$, we have

$$
0 \leq \int_{0}^{1} G(t, s) f(s, 0) d s=F 0,
$$

and so $\alpha(0, F 0) \geq 1$. Now, let $x_{n} \rightarrow x \in X$ and $\alpha\left(x_{n}, x_{n+1}\right) \geq 1$ for all $n \in \mathbb{N}_{0}$. Then, $x_{n} \preceq x_{n+1}$ and so $x_{n}(t) \leq x_{n+1}(t)$ for all $t \in I$. Hence, $x_{n}(t) \leq x(t)$ for all $t \in I$ and $n \in \mathbb{N}_{0}$, that is, $x_{n} \preceq x$ for all $n \in \mathbb{N}_{0}$. This implies that $\alpha\left(x_{n}, x\right) \geq 1$. Also, thanks to $x \preceq x$ for all $x \in X$, we get $\alpha(x, x) \geq 1$. This means that $X$ is $\alpha$-regular.

On the other hand, if $\alpha\left(x_{n}, x_{n+1}\right) \geq 1$ for all $n \in \mathbb{N}_{0}$, then $x_{n} \preceq x_{n+1}$ for all $n \in \mathbb{N}_{0}$. That is, the sequence $\left\{x_{n}\right\}$ is nondecreasing. Then, for all $m, n \in \mathbb{N}$ with $m<n$, we deduce that $x_{m} \preceq x_{n}$, and so $\alpha\left(x_{m}, x_{n}\right) \geq 1$. Thus, all the hypotheses of Corollary 2 are satisfied and so $F$ has a fixed point in $X$. Therefore, the problem (3.1) has a solution in $X$. Finally, let $u$ and $v$ be two solutions of (3.1). Then either $u \preceq v$ or $v \preceq u$. That is, $\alpha(u, v) \geq 1$ or $\alpha(v, u) \geq 1$. Thus, by the condition (iii) of Corollary 2 , the solution is unique. 
Example 2. Consider the following two-point boundary value problem of the second order differential equation:

$$
\left\{\begin{array}{c}
-\frac{d^{2} x}{d t^{2}}=\lambda t+\frac{x}{2+\sin t \frac{\pi}{2}}, \quad t \in I, \lambda \geq 0 \\
x(0)=x(1)=0
\end{array}\right.
$$

Let

$$
f(t, x)=\lambda t+\frac{x}{2+\sin t \frac{\pi}{2}}, \quad t \in I, \lambda \geq 0 .
$$

Then, it can easily see that $f(t, x)$ is continuous, nondecreasing with respect to the second variable and $f(t, 0) \geq 0$ for all $t \in I$. Further for $x \preceq y$, we have

$$
[f(t, x)-f(t, y)]^{2}=\left[\frac{x}{2+\sin t \frac{\pi}{2}}-\frac{y}{2+\sin t \frac{\pi}{2}}\right]^{2} \leq\left(\frac{x-y}{2}\right)^{2} .
$$

Letting $\theta(t)=\frac{t^{2}}{4}$ for all $t \in \mathbb{R}^{+}$and using Theorem 5, we deduce that the boundary value problem (3.4) has a unique solution.

\section{REFERENCES}

[1] R. Agarwal, D. O'Regan, and M. Sambandham, "Random and deterministic fixed point theory for generalized contractive maps," Appl. Anal., vol. 83, pp. 711-725, 2004, doi: 10.1080/00036810410001657206.

[2] S. Banach, "Sur les opérations dans les ensembles abstraits et leur application aux équations intégrales," Fundam. Math., vol. 3, pp. 133-181, 1922.

[3] M. Berzig and M. Rus, "Fixed point theorems for $\alpha$-contractive mappings of Meir-Keeler type and applications," Nonlinear Anal. Model. Control, vol. 19, no. 2, pp. 178-198, 2014.

[4] D. Boyd and J. Wong, "On nonlinear contractions," Proc. Amer. Math. Soc., vol. 20, no. 2, pp. $458-465,1969$.

[5] L. Ciric, "Fixed points for generalized multi-valued mappings," Mat. Vesnik, vol. 9, no. 24, pp. 265-272, 1972.

[6] G. Durmaz, G. Minak, and I. Altun, "Fixed point results for $\alpha-\psi$-contractive mappings including almost contractions and applications," Abstr. Appl. Anal., vol. 2014, p. 10 pp., 2014, doi: $10.1155 / 2014 / 869123$.

[7] H. Isik, B. Samet, and C. Vetro, "Cyclic admissible contraction and applications to functional equations in dynamic programming," Fixed Point Theory Appl., vol. 2015, no. 163, pp. 1-19, 2015, doi: 10.1186/s13663-015-0410-6.

[8] G. Jungck and B. Rhoades, "Fixed points for set valued functions without continuity," Indian J. Pure Appl. Math., vol. 29, pp. 227-238, 1998.

[9] R. Kannan, "Some results on fixed points," Bull. Calcutta Math. Soc., vol. 10, pp. 71-76, 1968.

[10] M. Khan, M. Swaleh, and S. Sessa, "Fixed point theorems by altering distances between the points," Bull. Aust. Math. Soc., vol. 30, no. 1, pp. 1-9, 1984, doi: 10.1017/S0004972700001659.

[11] P. Kumam, C. Vetro, and F. Vetro, "Fixed points for weak $\alpha-\psi$-contractions in partial metric spaces," Abstr. Appl. Anal., vol. 2013, p. 9 pp., 2013, doi: 10.1155/2013/986028.

[12] V. La Rosa and P. Vetro, "Common fixed points for $\alpha-\psi-\varphi$-contractions in generalized metric spaces," Nonlinear Anal. Model. Control, vol. 19, no. 1, pp. 43-54, 2014. 
[13] A. Latif, H. Isik, and A. Ansari, "Fixed points and functional equation problems via cyclic admissible generalized contractive type mappings," J. Nonlinear Sci. Appl., vol. 9, no. 3, pp. 1129-1142, 2016.

[14] J. Matkowski, "Fixed point theorems for mappings with a contractive iterate at a point," Proc. Amer. Math. Soc., vol. 62, no. 2, pp. 344-348, 1977, doi: 10.1090/S0002-9939-1977-0436113-5.

[15] J. Nieto and R. Rodríguez-López, "Contractive mapping theorems in partially ordered sets and applications to ordinary differential equations," Order, vol. 22, pp. 223-239, 2005, doi: 10.1007/s11083-005-9018-5.

[16] D. Patel, T. Abdeljawad, and D. Gopal, "Common fixed points of generalized Meir-Keeler $\alpha-$ contractions," Fixed Point Theory Appl., vol. 2013, no. 260, p. 16 pp., 2013, doi: 10.1186/16871812-2013-260.

[17] A. Ran and M. Reurings, "A fixed point theorem in partially ordered sets and some applications to matrix equations," Proc. Amer. Math. Soc., vol. 132, pp. 1435-1443, 2004.

[18] S. Reich, "Kannan's fixed point theorem," Boll. Unione Mat. Ital., vol. 4, no. 4, pp. 1-11, 1971.

[19] P. Salimi, C. Vetro, and P. Vetro, "Fixed point theorems for twisted $(\alpha, \beta)-\psi$-contractive type mappings and applications," Filomat, vol. 27, no. 4, pp. 605-615, 2013, doi: 10.2298/FIL1304605S.

[20] B. Samet, C. Vetro, and P. Vetro, "Fixed point theorems for $\alpha-\psi$-contractive type mappings," Nonlinear Anal., vol. 75, pp. 2154-2165, 2012, doi: 10.1016/j.na.2011.10.014.

[21] Y. Su, "Contraction mapping principle with generalized altering distance function in ordered metric spaces and applications to ordinary differential equations," Fixed Point Theory Appl., vol. 2014, no. 227, pp. 1-15, 2014, doi: 10.1186/1687-1812-2014-227.

Authors' addresses

Huseyin Isik

Department of Mathematics, Faculty of Science, Gazi University, 06500-Teknikokullar, Ankara, Turkey, Department of Mathematics, Faculty of Science and Arts, Muş Alparslan University, Muş 49100, Turkey

E-mail address: isikhuseyin76@gmail.com

\section{Duran Turkoglu}

Department of Mathematics, Faculty of Science, Gazi University, 06500-Teknikokullar, Ankara, Turkey

E-mail address: dturkoglu@gazi.edu.tr 